\section{D.3 METHODS OF ESTIMATING LIFETIME OCCUPATIONAL EXPOSURE IN THE GENERAL POPULATION, BASED ON JOB-EXPOSURE MATRICES}

\begin{abstract}
'Marie Houot*, 1,2Julie Homère, ${ }^{1,2}$ Hélène Goulard, 'Loïc Garras, 'Laurène Delabre, 1,2,3 Matgéné workgroup, ${ }^{1,3}$ Corinne Pilorget. 'Santé Publique France, the French National Public Health Agency, Saint-Maurice, France; ${ }^{2}$ University of Bordeaux, Occupational Health Team (Essat) Inserm UMR 1219, Bordeaux, France; ${ }^{3}$ University Claude Bernard Lyon1, IFSTTAR, UMRESTTE, UMR T_9405, Lyon, France
\end{abstract}

\subsection{6/OEM-2019-EPI.129}

Objectives To estimate proportion of pathologies attributable to occupational exposure, lifetime occupational exposure prevalence (LOEP) and relative risk are necessary. LOEP estimates are commonly used but often estimated with different methods. The method choice and the impact on estimates are rarely discuss in the literature. This study present and discuss the most widely used means of estimating LOEP and their respective impacts on estimates.

Methods A sample of individuals representative of the French population from 2007 was linked with four Matgéné jobexposure matrices: flour dust, cement dust, silica dust and benzene. LOEP and the 95\% confidence interval were estimated using five methods: the maximum exposure probability during the career (Method 1), four using individual exposure probabilities, three of which subdivide careers into job-periods (Methods 2-4) and one which subdivides them into job-years (Method 5). To quantify differences between methods, percentage of variation were calculated for prevalence values on Methods 2 to 5 versus Method 1 .

Results For each agent, LOEP estimated from the maximum probability during the career (Method 1) was consistently lower than prevalence taking account of job-periods or jobyears. LOEP on Method 1 for flour dust, cement dust, silica dust and benzene were respectively 4.4\%-95\% CI [4.0-4.7], $4.3 \%$ [3.9-4.6], 6.1\% [5.7-6.5] and 3.9\% [3.6-4.2]. Percentage of variation ranged from $0 \%$ to $25.0 \%$ for flour dust, from $11.6 \%$ to $55.8 \%$ for cement dust, from $11.5 \%$ to $49.1 \%$ for silica dust and from $0 \%$ to $53.8 \%$ for benzene.

Conclusions The present study provides a description of several LOEP estimation methods in the general population based on job-exposure matrices. It specifies the strong and weak points of each of the five chosen methods. For health monitoring purposes, LOEP should be reported as intervals, with low and high estimates obtained on different methods using job-periods (Methods 2-4).

\section{D.4 EXPOSURE PROFILES OF WORKERS IN INDIUM-TIN OXIDE POWDER MANUFACTURING, TARGET MANUFACTURING AND RECYCLING FACTORIES IN TAIWAN}

\begin{abstract}
${ }^{1}$ Yuan-Ting Hsu*, ${ }^{3}$ Ting-Yao Su, ${ }^{1}$ Hui-Yi Liao, ${ }^{4}$ Yu-Chieh Kuo, 'Saou-Hsing Liou. ${ }^{1}$ National Institute of Environmental Health Sciences, National Health Research Institutes, Miaoli County, Taiwan; ${ }^{2}$ Graduate Institute of Life Sciences, National Defense Medical Center, Taipei City, Taiwan; ${ }^{3}$ Division of Occupational Hazards Assessment, Institute of Labor, Occupational Safety and Health, Ministry of Labor, New Taipei City, Taiwan; ${ }^{4}$ Department of Environmental and Occupational Health, National Cheng Kung University, Tainan City, Taiwan
\end{abstract}

\subsection{6/OEM-2019-EPI.130}

We aimed to investigate indium exposure profiles and the relationship between ambient exposure and internal dose of indium among workers with different job characteristics in order to improve the work environment and protect workers from overexposure.

We recruited 329 workers from indium-tin oxide (ITO) powder and target manufacturing and recycling factories. The workers were categorized into six groups, as powder, ITO target, bonding, processing, recycling process and administration department as reference group. Field and personal air sampling were performed to monitor indium concentrations of work environments and breathing zones of workers. Cumulative exposure were evaluated by respirable dust concentrations in personal sampling, exposure duration and work duration. Plasma indium (P-In), urine indium (U-In) and U-In adjusted for creatinine (U-In/creatinine) were used as internal dose.

One-fourth of air indium concentrations of ITO manufacturing and recycling factories were exceeded permissible exposure limit (PEL) in Taiwan. Thirty-six percent of workers in this study exposed to unacceptable airborne concentration of indium. Over one-fifth of workers had P-In higher than Japanese biological exposure index (BEI) of $3 \mu \mathrm{g} / \mathrm{L}$. After adjusting for potential confounders, significant positive were found between indium cumulative exposure and P-In $(\beta=0.56$, $\mathrm{p}<0.001)$, U-In $(\beta=0.38, \quad \mathrm{p}<0.001)$, and U-In/creatinine $(\beta=0.34, p<0.001)$ in bonding process. A significant positive were found between indium cumulative exposure and P-In $(\beta=0.53, p=0.003)$, U-In $(\beta=0.39, p=0.047)$ and U-In/creatinine $(\beta=0.34, p=0.01)$ in processing process.

We suggest that U-In was an useful biomarker to assess indium exposure of indium manufacturing workers. The distribution and elimination of indium differed by its chemical form, which lead to characterization of the chemical form of indium is important for biomonitoring. Notably, although workers were exposed to indium below PEL, P-In still exceeded Japanese BEI. An appropriate exposure index need to be specified.

\section{D.5 CONSTRUCTION OF FINNISH ISCO-88 JOB EXPOSURE MATRIX: EXAMINATION OF DATASET WITH TWO DIFFERENT CLASSIFICATION OF OCCUPATIONS IN CONSECUTIVE CENSUSES}

Markku Sallmén*, Sanni Uuksulainen. Finnish Institute of Occupational Health, Helsinki, Finland

\subsection{6/OEM-2019-EPI.131}

Finnish Job exposure matrix (FINJEM) assesses occupational exposure for 84 factors in 311 FINJEM occupations. Finnish version of ISCO-88 International Standard Classification of Occupations 1988 (F-ISCO-88) occupational codes $(n=445)$, used in population censuses from 1995 to 2009, often split into more than one FINJEM code. We describe the construction of a crosswalk between F-ISCO- 88 codes and FINJEM codes and the resulting F-ISCO88 job exposure matrix (FISCO-88-JEM).

In total, we found 1144 conversion candidate pairs from two sources: 1) Statistics Finland crosswalks from three sequential classification of occupations (2001-1997, 1997-1987, 1987-1980) combined with crosswalk between the classification of occupations in 1980 and FINJEM, and 2) preliminary expert judgement-based conversion of F-ISCO-88-FINJEM.

We counted frequencies for all 9900 F-ISCO88 (in 1995) and FINJEM (1990) occupational code pairs from a study of 